\title{
First-Principles Theory of Correlated Transport through Nanojunctions
}

\author{
A. Ferretti, ${ }^{1}$ A. Calzolari, ${ }^{1}$ R. Di Felice, ${ }^{1}$ F. Manghi, ${ }^{1}$ M. J. Caldas, ${ }^{2}$ M. Buongiorno Nardelli, ${ }^{3,4}$ and E. Molinari ${ }^{1}$ \\ ${ }^{1}$ INFM National Center on nanoStructures and bioSystems at Surfaces $\left(S^{3}\right)$ \\ and Dipartimento di Fisica, Università di Modena e Reggio Emilia, 41100 Modena, Italy \\ ${ }^{2}$ Instituto de Física, Universidade de São Paulo, 05508-900 São Paulo, Brazil \\ ${ }^{3}$ Department of Physics, North Carolina State University, Raleigh, North Carolina 27695, USA \\ ${ }^{4}$ CCS-CSM, Oak Ridge National Laboratory, Oak Ridge, Tennessee 37831, USA
}

(Received 10 May 2004; published 22 March 2005)

\begin{abstract}
We report the inclusion of electron-electron correlation in the calculation of transport properties within an $a b$ initio scheme. A key step is the reformulation of Landauer's approach in terms of an effective transmittance for the interacting electron system. We apply this framework to analyze the effect of shortrange interactions on Pt atomic wires and discuss the coherent and incoherent correction to the mean-field approach.
\end{abstract}

DOI: 10.1103/PhysRevLett.94.116802

One of the most pressing problems in nanotechnology is the need for recasting all the knowledge about mesoscopic transport physics into the fully quantum mechanical limit appropriate for atomic-scale phenomena. In the case of electronic and transport properties of atomic and molecular conductors, we must address at the microscopic level both the chemical complexity of the conductor and the complexity of the interactions between the different components of an extended open system.

The combination of Green's function (GF) methods with a density functional theory (DFT) description of electronic states has become a standard approach to study charge transport at the nanoscale [1-5]. However, care must be taken in comparing the computed transport characteristics to experiments [6]. Indeed, some important featuressuch as electron correlations, dissipation, decoherence, and temperature effects - are at the moment partly or fully neglected. These deficiencies become more and more crucial when the dimensions of at least one part of the system reach a confinement situation where, for instance, electronelectron $(e-e)$ interactions may become dominant. Recent observations of the Kondo effect and Coulomb blockade in molecules connected to external electrodes $[7,8]$, and in nanotubes with magnetic impurities [9] or size confinement [10], indicate that correlations do play an important role in the mechanism of charge transport in nanodevices. Whereas much effort [6,11-14] has been directed to studying other aspects of the transport problem, a standard approach to include correlation effects does not yet exist. One may expect that some of the difficulties in the interpretation of transport experiments on simple atomic chains and individual molecules could be ascribed to the neglect or mistreatment of correlations.

In this Letter we develop a new method for the ab initio computation of quantum transport in the strong correlation regime, and then apply it to specifically address the effect of $e$-e interactions on electronic transport through atomicscale conductors. Following closely Meir and Wingreen [15], we derive a Landauer-like expression for the current
PACS numbers: 73.63.-b, 71.28.+d, 72.10.-d

through a correlated conductor between uncorrelated leads. Short-range $e-e$ interactions in the conductor are described through the three-body scattering (3BS) formalism [16,17]. The method is implemented through the use of "maximally localized" Wannier functions (MLWF) [5,18]. A particularly well-adapted system for gauging the relevance of $e$-e correlations is a late-transition-metal atomic chain with an uncorrelated portion representing bulk leads $(e-e$ effects are negligible in the bulk) and a correlated portion mimicking the one-dimensional wire in a nanojunction. We apply our method to simulate a model platinum atomic wire of varying lengths. Our findings show a large suppression of the transmittance that we ascribe to the inclusion of $e-e$ elastic decoherence in the simulation. Moreover, the strong conductance reduction, also for small wire lengths, suggests that correlation plays an important role when studying transport properties of systems with localized electrons.

The system is modeled as three different regions, the left $(L)$ and right $(R)$ electrodes and a central conductor $(C)$. We express our operators in a localized basis set [19] which allows us to write the Hamiltonian and the GFs of the whole system as $3 \times 3$ block matrices defined on the basis in the $L, R$, and $C$ regions $\left[H_{x y}, G_{x y}(\omega)\right.$ where $x, y=$ $L, C, R]$.

The Hamiltonian reads

$$
H=\sum_{l l^{\prime} \in L \text { or } R} H_{l l^{\prime}} c_{l}^{\dagger} c_{l^{\prime}}+H_{\mathrm{int}}+\sum_{\substack{l \in L \text { or } R \\ i \in C}}\left[H_{l i} c_{l}^{\dagger} d_{i}+\text { H.c. }\right] \text {, }
$$

where $c_{l}$ and $c_{l}^{\dagger}\left(d_{i}\right.$ and $\left.d_{i}^{\dagger}\right)$ are the one-electron annihilation and creation operators in the leads (conductor). In the above expression, the first term describes the $L$ and $R$ leads, $H_{\text {int }}$ the conductor, and the last term the coupling of the conductor with the $L$ and $R$ leads. We stress that the leads and the coupling Hamiltonian are noninteracting and all the $e-e$ interaction is restricted to the conductor.

From the continuity equation for the steady-state current in the system and using the Keldysh nonequilibrium GFs 
formalism [20], the following expression for the current [15] is derived:

$$
I=\frac{2 e i}{h} \int d \omega \operatorname{Tr}\left\{\left[\Sigma_{L}^{<}-\Sigma_{R}^{<}\right] A_{C}+\left[\Gamma_{L}-\Gamma_{R}\right] G_{C}^{<}\right\} .
$$

Here $A_{C}=i\left[G_{C}^{r}-G_{C}^{a}\right]$ is the spectral function and $G_{C}^{r, a,<,>}$ are the (retarded, advanced, lesser, greater) GFs in the conductor. The trace should be taken on the conductor. The interaction between conductor and leads is described through the lead self-energies (SE's), defined as $\Sigma_{x}^{r, a,<,>}(\omega)=H_{C x} G_{x}^{r, a,<,>}(\omega) H_{x C}, \quad$ where $\quad x=L, R$. Finally, the $\Gamma_{L, R}$ terms in Eq. (2) are defined as the negative of twice the imaginary part of the retarded lead SE's, i.e., $\Gamma_{L, R}=i\left[\Sigma_{L, R}^{r}-\Sigma_{L, R}^{a}\right]$. Note also that $\Sigma_{L, R}^{<}=i f_{L, R} \Gamma_{L, R}$ and $\Sigma_{L, R}^{>}=-i\left(1-f_{L, R}\right) \Gamma_{L, R}$, where $f_{L}$ and $f_{R}$ are the Fermi occupation functions for the left and right leads.

While in the noninteracting case the above expression brings us to the usual Landauer formula [15], in the presence of the interaction between electrons this is no longer true and further assumptions are needed. We here adopt the ansatz proposed by $\mathrm{Ng}$ [21] which relates $\Sigma_{C}^{<}\left(\Sigma_{C}^{>}\right)$to $\Sigma_{C}^{r, a}$, thus defining the statistics of energy levels, in the general out-of-equilibrium interacting case. The starting point is the assumption [22]

$$
\Sigma_{C}^{<,>}(\omega)=\Sigma_{0 C}^{<,>}(\omega) \Lambda(\omega),
$$

where $\Sigma_{0 C}^{<,>}=\Sigma_{L}^{<,>}+\Sigma_{R}^{<,>}$refer to the noninteracting case and include only the coupling with the leads, while the full $\Sigma_{C}^{<,>}$include also $e$-e interactions. $\Lambda(\omega)$, also called $A$ in other formulations [22], is determined by the identity $\Sigma_{C}^{>}-\Sigma_{C}^{<}=\Sigma_{C}^{r}-\Sigma_{C}^{a}$ leading to

$$
\Lambda(\omega)=\left[\Sigma_{0 C}^{r}(\omega)-\Sigma_{0 C}^{a}(\omega)\right]^{-1}\left[\Sigma_{C}^{r}(\omega)-\Sigma_{C}^{a}(\omega)\right] .
$$

Here the interacting SE's take the form

$$
\Sigma_{C}^{r, a}(\omega)=\Sigma_{L}^{r, a}(\omega)+\Sigma_{R}^{r, a}(\omega)+\Sigma_{\text {corr }}^{r, a}(\omega),
$$

where $\Sigma_{\text {corr }}^{r, a}$ account for the $e$ - $e$ interactions (while $\Sigma_{0 C}^{r, a}$ just drop this last term). Relations (3)-(5) are the key to relate Eq. (2) to a Landauer-like formula. In fact, following Eq. (6) of Ref. [22] and using $G_{C}^{<}=G_{C}^{r} \Sigma_{C}^{<} G_{C}^{a}$, it is possible to derive $G_{C}^{<}=i G_{C}^{r}\left[f_{L} \Gamma_{L}+f_{R} \Gamma_{R}\right] \Lambda G_{C}^{a}$ and therefore $G_{C}^{r}-G_{C}^{a}=-i G_{C}^{r}\left[\Gamma_{L}+\Gamma_{R}\right] \Lambda G_{C}^{a}$. These steps lead to the final expression for the current:

$$
I=\frac{e}{\hbar} \int \frac{d \omega}{2 \pi}\left[f_{L}-f_{R}\right] \operatorname{Tr}\left\{\Gamma_{L} G_{C}^{r} \Gamma_{R} \Lambda G_{C}^{a}\right\} .
$$

We note that the $e-e$ correlation plays a twofold role: it renormalizes the GFs, which should now be calculated for the interacting system, and also modifies the expression for the current, introducing the corrective factor $\Lambda(\omega)$. The quantity traced in Eq. (6), even if not a true transmittance across the scattering region due to the breakdown of Landauer's theory, still plays the same role as regards transport. For this reason we refer to it as an effective transmittance and compare it to the transmittance of the noninteracting case. In particular, since the imaginary part of the $e$ - $e$ self-energy exactly vanishes at the Fermi energy $\left(E_{\mathrm{F}}\right)$, the correction $\Lambda\left(\omega=E_{\mathrm{F}}\right)$ becomes the identity operator and the effective transmittance computed at $E_{\mathrm{F}}$ is proportional to the conductance at zero temperature, provided that the SE's are calculated for the interacting system.

An accurate evaluation of the effective transmittance needs to be based on (i) a good description of the noninteracting system, and (ii) the calculation of the $e-e$ correlated SE to include many-body effects arising from the interaction in the conductor. The first problem is solved by exploiting a recent methodology for the $a b$ initio calculation of the transmittance in the coherent transport regime [5]. The ground state of the mean-field system is described within the local density approximation (LDA) to DFT, using norm-conserving pseudopotentials and a planewave basis set [23]. The geometry of the $L-C-R$ nanojunction are as in Ref. [1]. To obtain a consistent description of the system in a real-space localized basis set, the MLWF's are computed both for the leads and for the conductor [24]. The details of this transformation and its application to electronic transport are described elsewhere $[5,18]$. We note that the basis change from Bloch eigenvectors to MLWF's preserves orthonormality and completeness in the original Hilbert subspace, thereby avoiding typical problems arising very often for other localized basis functions. The same features allow us to use a minimal basis set for computing transport properties, while employing the system independent plane-wave basis set for the DFT calculation.

To discuss the inclusion of $e$ - $e$ correlations, we first need to define the interaction Hamiltonian in Eq. (1). We focus on the short-range $e-e$ interaction for two main reasons. On the one hand, this regime is characterized by strong deviations from the noninteracting behavior, for instance, in terms of quasiparticle lifetimes (which include elastic decoherence in the transport formalism). On the other hand, it allows us to adopt an Anderson-like form [25] of the interaction which is suitable for the localization in the conductor region only, as required by our approach.

In this work the $e$-e self-energy $\Sigma_{\text {corr }}^{r}$ is computed using a nonperturbative approach based on an effective Anderson Hamiltonian, whose $U$ Coulomb integrals could be either calculated ab initio [26] or used as adjustable input parameters. It is solved by means of a configuration interaction scheme with up to three bodies (3BS) added to the noninteracting Fermi sea: two (one) electron(s) and one (two) hole(s) for conduction (valence) states. This method has been successfully applied to describe photoemission experiments on strongly correlated systems [17]. The 3BS self-energy is formally given [17] as a sum over projectors onto atomic states (those with non-negligible $U$ integrals) and thus can be properly localized in the conductor.

We now come to the model system: a Pt atomic wire of varying lengths, where the correlation is switched on only on a finite number $N_{C}$ of atoms. It is worth noting that late $5 d$ transition-metal atomic wires have been demonstrated 
in break-junction experiments [27]. Moreover, in the case of $\mathrm{Pt}$, correlation effects are expected to strongly increase their importance in passing from the bulk leads to the confined wire as an effect of the reduced dimensionality. Therefore, our model system, while neglecting the full complexity of the wire-lead interfaces, retains the basic geometric construct of a correlated wire between uncorrelated leads in a Pt break junction. Hence, because the interface-induced effects are already well known [27], this model allows us to elucidate the effects of correlation and decoherence in nanojunctions. The inclusion of both correlation and interface effects is discussed below with a further example.

The setup is described in Fig. 1(a). For region $C$ we consider a supercell containing 11 atoms with an interatomic separation of $3.0 \AA$, reasonable for experiments under stretching (such as break junctions [27]). The same geometry is adopted for the leads, which are modeled as semi-infinite wires treated at the single-particle level. Since the $s d$ bands of the Pt chain [Fig. 1(b)] form an isolated subset [18], we can extract a manifold of MLWF's which span the same subspace. As an example, a particular wave function well localized within two bond lengths is shown in the inset of Fig. 1(c). This MLWF subspace allows us to reproduce with good accuracy states more than $2 \mathrm{eV}$ above the Fermi energy, enough to describe transport properties in this system where $p$ orbitals are expected to play a negligible role. Figure 1(c) shows the computed transmittance for the noninteracting Pt chain, which counts, for each energy, the number of channels (bands) available for charge transport, leading to a zero temperature conductance of $3 G_{0}\left(G_{0}=2 e^{2} / h\right)$.

In Figs. 2(a) and 2(b) we report the spectral function projected on the interacting region and the effective transmittance for a chain with three correlated atoms $(U=$ $2.0 \mathrm{eV}$ ). The many-body spectral function shows a split-

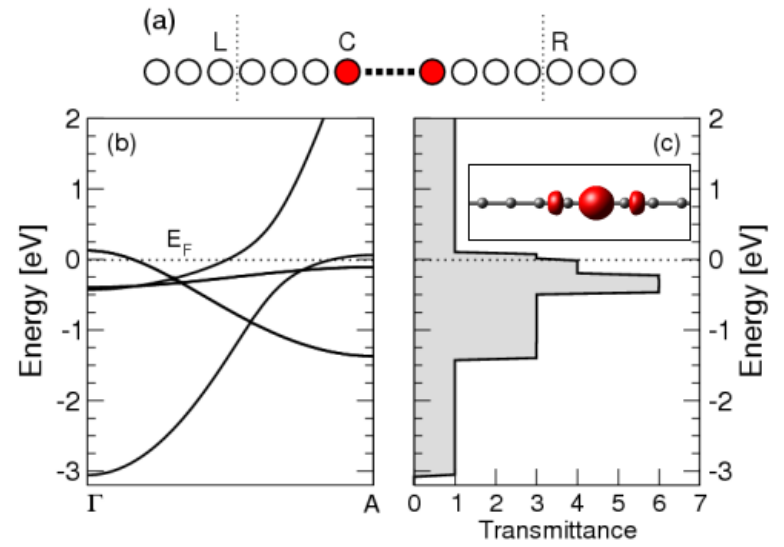

FIG. 1 (color online). (a) System layout divided in the leads and conductor regions. Open (full) circles are uncorrelated (correlated) Pt atoms. (b) Band structure of the Pt infinite chain. $A$ is the edge of the one-dimensional Brillouin zone. (c) Transmittance of the noninteracting conductor region. The inset shows the isosurface plot of a computed MLWF. ting of the $d$ bands and a slight upward energy shift, which is consistent with our short-range interaction picture based on the physics of the Anderson Hamiltonian. The transmittance is strongly suppressed by the inclusion of correlation [Fig. 2(b)], which is particularly effective in the hole region. This can be understood considering that on-site correlation arises from the strongly localized $\mathrm{Pt} d$ orbitals that are largely occupied and produce major features below $E_{\mathrm{F}}$.

The introduction of $e$ - $e$ interactions leads to quasiparticles characterized by energy and lifetime (finite broadening of their spectral features) formally accounted for by the Hermitian $(H)$ and the anti-Hermitian $(A)$ parts of the SE operator. Note that in more common correlation treatments, such as LDA + U, the SE is Hermitian and lifetimes are consequently neglected [28]. Although these components must obey analytic constraints [17], we analyze them separately to highlight their very effect on transport. First of all, we divide the SE operator in $H$ and $A$ contributions, $\Sigma_{\text {corr }}=\Sigma_{H}+\Sigma_{A}$, where

$$
\Sigma_{H, A}(\omega)=\frac{1}{2}\left[\Sigma(\omega) \pm \Sigma^{\dagger}(\omega)\right] .
$$

In Figs. 2(c),2(d) and 2(e),2(f) we show our results when using $\Sigma_{H}$ or $\Sigma_{A}$, respectively, instead of the full correlation SE from 3BS. By definition $\Sigma_{A}$ vanishes at $E_{\mathrm{F}}$; thus the only contribution to the zero temperature conductance comes from the $H$ part of $\Sigma$. Moreover, at energies different from $E_{\mathrm{F}}$ the major quenching factor on the transmittance is due to the $A$ part of $\Sigma$, while its effect on the spectral function is just a slight broadening of the main peaks. Note that the decrease of the effective transmittance due to $\Sigma_{H}$ is related to energy misalignment of channels between the conductor and the leads, while that due to $\Sigma_{A}$ accounts for $e-e$ scattering. Our results thus indicate that
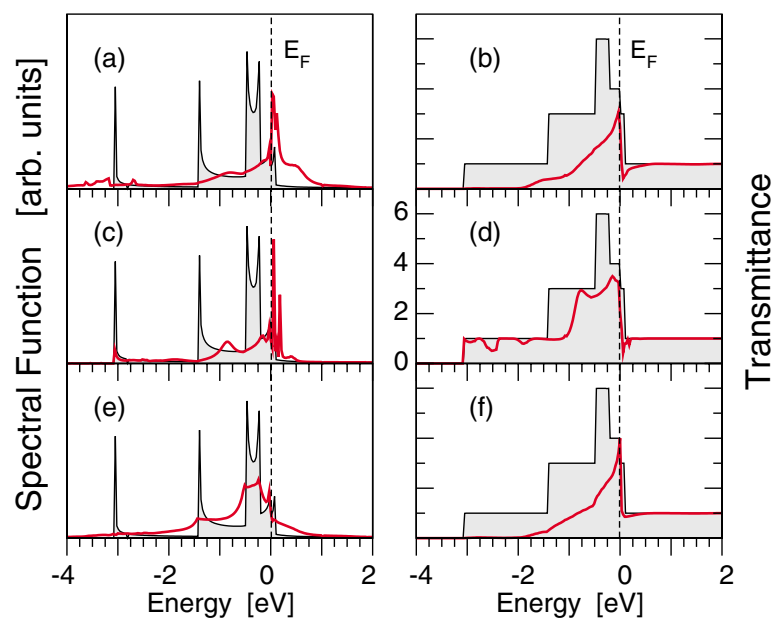

FIG. 2 (color online). Curves: Spectral function projected on the interacting region (left) and effective transmittance (right) for the case of three correlated atoms in the Pt chain. Shaded areas: mean-field reference results. (a),(b) The results obtained with the total correlation SE $\Sigma_{\text {corr }}=\Sigma_{3 B S}$. The (c),(d) [(e),(f)] plots are obtained using only the Hermitian [anti-Hermitian] SE $\Sigma_{H}\left[\Sigma_{A}\right]$. 


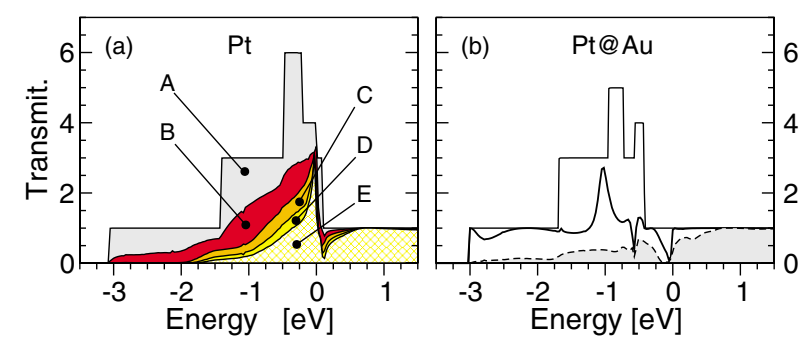

FIG. 3 (color online). (a) Effective transmittance against energy for variable number of correlated atoms in the chain: $A$ is the reference mean-field bulk transmittance. $N_{C}=1,3,5,7$ in B, C, D, E. (b) Effective transmittance for a Au-Pt-Au junction (three Pt atoms). Solid lines: mean-field description of an infinite $\mathrm{Au}$ chain (thin line) and of the Au-Pt-Au interface (thick line). Shaded area: interface treatment including correlation.

$e-e$ scattering plays a fundamental role in suppressing effective transmittance for systems with strong short-range correlation. This effect should be hidden in the low temperature conductance but be evident in the $I-V$ characteristics [Eq. (6)]: a suggestion for further experimental analysis.

Figure 3(a) shows the effective transmittance curves for various numbers of correlated atoms $N_{C}$ and shows how the effective transmittance decreases with increasing $N_{C}$. A direct analysis of the effective transmittance at $E_{\mathrm{F}}$ reveals also a rapid decreasing zero-temperature conductance. This finding suggests that the effect of on-site correlation should be experimentally measurable also for short atomic chains. In order to compare the effects on the transmittance due to interfaces with those due to correlation, we also studied the case of a Au-Pt-Au junction with the conductor formed of three correlated Pt atoms and the leads of semiinfinite mean-field $\mathrm{Au}$ chains (we tested that electronic correlation is ineffective in Au even at low dimensionality). The geometry is the same as in Fig. 1(a). We treat this interface first at a mean-field level and then fully include the $e-e$ interactions on Pt. Figure 3(b) reports the results: the trends due to the inclusion of correlation are the same as before, also for an interface between two different materials. Again, we find that the main role is played by the imaginary part of the $e-e \mathrm{SE}$, consistently with the results for Pt chains. This finding a posteriori validates the choice of the Pt wire as a good model system.

In conclusion, we derived a generalized Landauer-like expression for the current [Eq. (6)] and the conductance in the presence of $e-e$ interactions. The formalism is suitable for a fully $a b$ initio implementation that we realized using a basis set of maximally localized Wannier functions for the $\mathrm{GFs}$ and the 3BS formalism for the $e-e$ self-energy. We applied the method to a finite Pt wire and found a renormalization of the conductance and a strong quenching of the transmittance as a consequence of the $e$-e scattering. Our results suggest that the inclusion of electron correlation, for systems with strong short-range interactions, is important for an accurate description of transport if dimensionality effects strongly influence the electronic structure, as in the atomic chains studied here.

We acknowledge illuminating discussions with C.A. Rozzi and V. Bellini. Funding was provided by the EC through Project No. IST-2001-38951 and TMR network "Exciting," by INFM through "Commissione Calcolo Parallelo" and by MIUR (Italy) through "FIRBNOMADE." M.B.N. and A.C. acknowledge the Petroleum Research Fund of the ACS. M. B. N. also acknowledges the Mathematical, Information and Computational Sciences Division, Office of Advanced Scientific Computing Research of the U.S. DOE under Contract No. DE-AC05-00OR22725 with UT-Battelle. M. J.C. acknowledges support from INFM $\mathrm{S}^{3}$, Italy, FAPESP and CNPq, Brazil.

[1] M. Buongiorno Nardelli et al., Phys. Rev. B 64, 245423 (2001).

[2] J. Taylor et al., Phys. Rev. B 63, 245407 (2001).

[3] M. Brandbyge et al., Phys. Rev. B 65, 165401 (2002).

[4] Y. Xue et al., Chem. Phys. 281, 151 (2002).

[5] A. Calzolari et al., Phys. Rev. B 69, 035108 (2004).

[6] F. Evers et al., Phys. Rev. B 69, 235411 (2004).

[7] W. Liang et al., Nature (London) 417, 725 (2002).

[8] J. Park et al., Nature (London) 417, 722 (2002).

[9] T. W. Odom et al., Science 290, 1549 (2000).

[10] P. Jarillo-Herrero et al., Nature (London) 429, 389 (2004).

[11] M. Di Ventra and S. T. Pantelides, Phys. Rev. B 61, 16207 (2000).

[12] D. S. Kosov, J. Chem. Phys. 119, 1 (2003).

[13] N. T. Maitra et al., Phys. Rev. B 68, 045109 (2003).

[14] P. Delaney and J.C. Greer, Phys. Rev. Lett. 93, 036805 (2004).

[15] Y. Meir and N.S. Wingreen, Phys. Rev. Lett. 68, 2512 (1992).

[16] C. Calandra and F. Manghi, Phys. Rev. B 50, 2061 (1994).

[17] F. Manghi et al., Phys. Rev. B 56, 7149 (1997).

[18] N. Marzari and D. Vanderbilt, Phys. Rev. B 56, 12847 (1997).

[19] S. Datta, Electronic Transport in Mesoscopic Systems (Cambridge University Press, Cambridge, U.K., 1995).

[20] H. Haug and A.-P. Jauho, Quantum Kinetics in Transport and Optics of Semiconductors (Springer, Berlin, 1996).

[21] T.-K. Ng, Phys. Rev. Lett. 76, 487 (1996).

[22] N. Sergueev et al., Phys. Rev. B 65, 165303 (2002).

[23] S. Baroni, A. Dal Corso, S. de Gironcoli, and P. Giannozzi, http://www.pwscf.org, 2001.

[24] A. Calzolari, C. Cavazzoni, N. Marzari, and M. Buongiorno Nardelli, WANT code, http://www.wanniertransport.org, 2004; see also Ref. [5].

[25] G. D. Mahan, Many-Particle Physics (Plenum, New York, 1981).

[26] M. Springer and F. Aryasetiawan, Phys. Rev. B 57, 4364 (1998).

[27] N. Agraït et al., Phys. Rep. 377, 81 (2003).

[28] A. Ferretti et al. (unpublished). 